\title{
Updated review: drug-resistant epilepsy and presurgical evaluation of epilepsy surgery
}

\author{
TL Poon *, Colin HT Lui, Iris Chan, Deyond YW Siu; Subcommittee on the Consensus Statement of The \\ Hong Kong Epilepsy Society
}

- A video clip showing insertion of depth electrode is available at $<w w w$. hkmj.org>

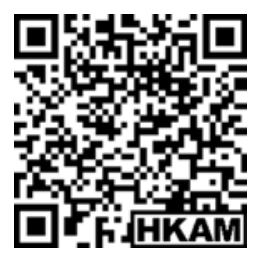

This article was published on $27 \mathrm{Nov}$ 2018 at www.hkmj.org.

\section{A B S T R A C T}

Epilepsy is defined as drug-resistant after failure of two adequate trials of appropriately chosen and administered antiepileptic drugs. Approximately $30 \%$ of patients with epilepsy have drug-resistant epilepsy. Reasons for treatment failure include failure to recognise epilepsy syndrome, poor drug compliance, and lifestyle factors. Patients with drugresistant epilepsy should be encouraged to have early referral to a tertiary epilepsy centre for presurgical evaluation. Comprehensive neurophysiology, structural neuroimaging, neuropsychological, and psychiatric assessments are regarded as essential for determining suitability for epilepsy surgery. Epilepsy surgery, whether resection, disconnection, or neuromodulation, should be recommended only after multidisciplinary consensus agreement based on these assessments.

\section{Background}

Patients with epilepsy whose seizures do not respond successfully to antiepileptic drug therapy are considered to have drug-resistant epilepsy (DRE). Prior equivalent terms include medically intractable epilepsy or pharmacoresistant epilepsy. Among patients with epilepsy, those with DRE have the greatest burden of epilepsy-related disabilities and of health care expenses.

In 2010, the task force of the International League Against Epilepsy Commission on Therapeutic Strategies proposed a framework of two levels for defining DRE. ${ }^{1}$ The diagnosis of DRE usually requires failure of two adequate trials of appropriately chosen and administered antiepileptic drugs (sequential monotherapy or combined polytherapy). ${ }^{1,2}$ It is also important to consider the effect of seizure factors (frequency, severity, associated behavioural problem) on individual psychosocial well-being. This effect will influence the physicians' decision on drug options and the urgency of considering nonpharmacological therapy.

The important aspects of the clinical assessment of DRE are discussed in the sections below.

\section{Epidemiology}

A 2004 study estimated the prevalence of epilepsy among Hong Kong Chinese residents aged $\geq 15$ years at about 3 to 5.7 per 1000 population, or
Hong Kong Med J 2018;24:610-6

DOI: 10.12809/hkmj187285

${ }^{1}$ TL Poon *, FHKAM (Surgery), FRCS (SN) (Edin)

CHT Lui, FHKAM (Medicine)

${ }^{3}$ I Chan, PhD

${ }^{4}$ DYW Siu, FHKAM (Radiology)

for the Subcommittee on the Consensus Statement of The Hong Kong Epilepsy Society

${ }^{1}$ Department of Neurosurgery, Queen Elizabeth Hospital, Jordan, Hong Kong

Department of Medicine, Tseung Kwan O Hospital, Tseung Kwan O, Hong Kong

Department of Medicine, Queen Elizabeth Hospital, Jordan, Hong Kong

${ }^{4}$ Department of Diagnostic and Interventional Radiology, Kwong Wah Hospital, Yaumatei, Hong Kong

*Corresponding author: poontaklap@yahoo.com.hk

approximately 40000 Hong Kong residents in total. ${ }^{3}$ Approximately one third of these patients with epilepsy were expected to have DRE. ${ }^{3}$ A study in 2008 estimated the crude prevalence of active epilepsy and seizure disorder in Hong Kong to be 8.49 per 1000 population; therefore, the number of people in Hong Kong at that time with seizure disorder was approximately $62000 .^{4}$ A meta-analysis of international studies showed the point prevalence of epilepsy was 6.38 per 1000 population. ${ }^{5}$ Furthermore, a recent study regarding prevalence and incidence of epilepsy in the United States population showed that the overall age-adjusted prevalence of epilepsy was 8.5 per 1000 population. ${ }^{5}$ Previous studies have found that there is a treatment or referral gap of 20 years in the United States for patients with DRE. ${ }^{5,6}$

\section{Pathogenesis}

Prospective studies of patients with chronic epilepsy suggested that $70 \%$ to $80 \%$ of patients retain their status as either having intractable seizures or being in remission. ${ }^{7}$ In other words, around $20 \%$ of patients with seizure initially diagnosed as intractable will achieve seizure freedom in the long term. One proposed mechanism that might lead to intractability involves glial proliferation and dendritic sprouting with synaptic recognition in mesial temporal sclerosis. ${ }^{8}$ Paroxysmal depolarization shift refers to situations where the usual refractory period does not follow rapidly repeating action potentials at a cellular level, resulting in local high-frequency 
oscillations. ${ }^{9,10}$

Another compelling theory is the build-up of epileptic "neuronal network", via alternation in neuronal circuitry. ${ }^{11}$ A well-defined neuronal network example is the limbic network with sequential propagation path via the hippocampus, amygdala, lateral temporal neocortex, entorhinal cortex, medial thalamus, and frontal inferior lobes. The interest on neuronal network analysis in epilepsy had gained strength with the use of high-resolution recording techniques. Different brain functional regions are interconnected with specific neuronal networks; therefore, a seizure in one part will spread quickly in a typical oscillatory manner. ${ }^{11}$

\section{Clinical course}

A recent study, which adopted the International League Against Epilepsy DRE criteria, observed different patterns of disease progression in an incident cohort of DRE. ${ }^{12}$ The authors of that study observed that $30 \%$ of patients eventually developed DRE. They also found a long delay from disease onset to failure of second antiepileptic drug. This finding might give insights into the pathogenesis of DLE mentioned above.

In general, the mortality and morbidity of DRE is higher than that of seizure-free patients or patients with good seizure control. ${ }^{13-15}$ Even when seizures are infrequent, daily life and subjective wellbeing are impaired in patients with DLE, to various extents. ${ }^{16}$

\section{Classification}

Refractory epilepsy can be subdivided into temporal lobe epilepsy (TLE) and non-temporal lobe epilepsy. Depending on the clinical and radiological manifestations, TLE can be divided further into two distinct groups: mesial TLE and neocortical TLE. Both mesial TLE and neocortical TLE share similar pathological substrates; the primary difference is that mesial temporal sclerosis is found only in mesial TLE. ${ }^{17}$ Patients with TLE usually present with complex partial seizures, with or without generalised seizures, depending on the neuronal network involved. A minority of patients with TLE will became seizure free after repeated drug trials. ${ }^{18}$ However, most patients with TLE require surgical intervention. ${ }^{18}$

For patients with non-temporal lobe epilepsy, the clinical and radiological features are diverse and also depend on the underlying aetiologies or pathological substrates. In general, the seizure semiology is poorly defined and variability in the associated magnetic resonance imaging (MRI) abnormalities makes seizure focus localisation challenging. Usually, a concerted effort and multimodality investigations (in phase 1 of the presurgical evaluation) are required. ${ }^{19}$

\section{抗藥性癲㾫及癲㓕的術前評估 \\ 潘德立、呂曉東、陳詠詩、蕭容媛}

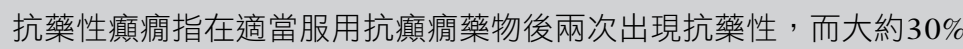

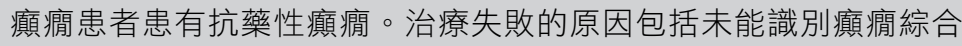
徵, 藥物依從性欠佳以及生活方式因素。應鼓勵患有抗藥性癲癇患者 及早轉介至三級癲瘉中心進行術前評估。綜合神經生理學、結構神 經成像、神經心理學和精神病評估能確定是否適合癲瘉手術的必要條 件。不論是切除性、分隔或神經調控癲痌手術, 應基於上述評估的多 學科共識協議後才進行。

\section{Approach after drug treatment failure}

Before patients with DRE are referred to a tertiary centre for preparation for surgical intervention, potential factors for treatment failure should be considered. For example, failure to recognise a generalised epilepsy syndrome can result in an inappropriate choice of first-line antiepileptic drug (eg, carbamazepine) that will aggravate seizures. Poor drug compliance and other lifestyle-related factors can also contribute to seizure recurrence. These factors are often considered as pseudoresistance. ${ }^{20-22}$

\section{Selection of eligible candidates}

The longer the delay between the onset of DRE and surgery intervention, the lower the chance of a seizure-free and improved psychosocial postoperative outcome. ${ }^{5}$ Therefore, timely referral is crucial for quality care of patients with DRE.

Before recruiting the patient, the first step is to identify the criteria that indicate an appropriate candidate. Only patients who meet all three of these eligibility criteria should be considered for inclusion: 1. The patient and their family understand and accept the surgical treatment and any potential risks;

2. The seizures are disabling despite adequate and appropriate drug trials; and

3. The available imaging and neurophysiological data are consistent with surgically remediable epileptic syndrome.

\section{Presurgical evaluations}

The first objective of presurgical evaluation is to identify the epileptogenic zone (EZ) by invasive or non-invasive modalities of investigations. More sophisticated or invasive approaches might be required, depending on the clarity of structural identifiable pathologies on neuroimaging, and on the link with the clinical semiology.

The second objective, after successful 
identification and location of the EZ, is to develop strategies to ensure that the lesion can be safely resected without significant physical or cognitive sequelae.

It is also pragmatic to interview the patient and their family and friends, in order to obtain all relevant history and risk factors or aetiological factors. The latter will also give insight into the prognosis of the epileptic disorder after surgical treatment. For example, a case of post-encephalitic epilepsy would be less suitable for surgical intervention. ${ }^{23}$

A multidisciplinary evaluation team is required, and investigations should include neurophysiological evaluation, structural neuroimaging, neuropsychological assessment, and psychiatric assessment.

\section{Neurophysiological evaluation}

The neurophysiological evaluation includes interictal and ictal electroencephalography (EEG) sampling, which can be attained by non-invasive or invasive means in a long-term recording manner.

Interictal EEG provides important information on the lateralisation or localisation of the EZ. This is particularly true in cases of TLE, in which a solely unilateral anterior temporal spike is a strong predictor of a seizure-free postoperative outcome. ${ }^{24}$ Unilateral mesial temporal sclerosis with bitemporal interictal epileptiform discharges may also be found. ${ }^{25}$ Short bursts of low-voltage, high-frequency oscillations are valuable in localising focal cortical dysplasia. ${ }^{26}$

Video EEG recordings capture habitual seizures and ictal EEG discharges. The lateralisation and localisation of the ictal onset zone can be deduced from analysis of an adequate number of these captured events. After combined analysis of ictal and interictal EEG data, the irritative and ictal onset zones can be estimated. ${ }^{27}$

Invasive recording is indicated when there is a hypothesised EZ that is not fully supported by the results of non-invasive diagnostic evaluations. These difficult scenarios are more common in cases of nonlesional epilepsy. ${ }^{28,29}$

\section{Structural neuroimaging}

Brain MRI is the fundamental yet most important investigation for presurgical evaluation. This is particularly true for certain epileptic disorders, such as TLE in which mesial temporal sclerosis is the pathological substrate. The MRI features of hippocampal sclerosis include hippocampal atrophy on T1-weighted MRI images, increased signal on T2-weighted MRI images or fluid-attenuated inversion recovery MRI sequences, and decreased signal on inversion recovery MRI sequences..$^{30,31}$ Detection of these abnormalities requires optimised imaging techniques, including angulated coronal sections obtained perpendicular to the long axis of the hippocampal structures.

For extratemporal substrates, MRI can also define hemimegaloencephaly, schizencephaly, and focal subcortical heterotopia. Focal cortical dysplasia is the most common developmental pathology in children with extratemporal lobe seizures, and there is an international classification to define the underlying histopathology and foretell the outlook of surgical success. ${ }^{32}$

Recently, 3T MRI systems have gained in importance for pre-surgical workup. Studies have shown that, for initially non-lesional cases scanned by a $1.5 \mathrm{~T}$ system with standard MRI brain protocol, more than half had new findings after rescanning by a 3T MRI system with multichannel phased-array coils. ${ }^{33,34}$

Diffusion tensor imaging and tractography can be used for fibre tracking and non-invasive structural network mapping and is an optional imaging sequence to aid presurgical trajectory planning. A recent study reported using diffusion tensor imaging for successful identification of significant diffusion abnormalities of tract sections in the ipsilateral dorsal fornix and in the contralateral parahippocampal white matter bundle in patients with poor postoperative seizure control. ${ }^{35}$ Although more studies are required to draw a definitive conclusion, these results may help to understand the mechanisms of postoperative persistent seizure. Diffusion tensor imaging has potential prognostic value in predicting operation outcome.

Despite the strengths of MRI, there are pathological substrates that go beyond the detection ability of MRI analysis. As a result, multi-modality imaging of the brain will come into play. Functional neuroimaging modalities, such as positron emission tomography, single photon emission computed tomography, functional MRI and magnetoencephalography, can be co-registered with MRI to give a more detailed structural-functional correlated imaging analysis. These imaging modalities can aid the localisation of EZ, but the sensitivity largely depends on the epileptic syndrome. Magnetoencephalography is indicated in both localization of EZ and delineation of the relationship between the index lesion and the surroundings. Magnetoencephalography-guided excisional surgery provides more precise excision in subtle or MRInegative cases, and in patients with multiple intracerebral lesions, such as cavernomata. ${ }^{36-38}$

\section{Neuropsychological assessment}

Neuropsychological tests rely on functional neuroanatomy, in which certain cognitive functions are ascribed to physical areas of the brain. The temporal lobe is associated with memory and language, with the left side representing verbal 
memory, and the right side representing visual memory. The frontal lobe is associated with executive actions and behaviour, and the posterior of the brain with perception and higher sensory faculties. It is controversial to state the prediction of postoperative cognitive outcome should be based on the side that was to be resected or the side that would remain following surgery.

The Wada test, first introduced in 1949, is used to determine cerebral language dominance. ${ }^{39}$ The Wada test is used to evaluate the risks of postoperative amnesia and task-specific memory deficits, to lateralise hemispheric dysfunction, and to predict postoperative seizure outcome. ${ }^{39}$ However, one study involving 145 patients showed that Wada test results were not predictive of outcome. ${ }^{40}$ Functional MRI is an alternate non-invasive method to determine cerebral language dominance. Functional MRI has been shown as eligible to replace Wada tests in the majority of patients with clearly lateralised language localisation; however, in patients who are agitated or mentally impaired and have bilateral functional MRI activations, Wada tests still provide additional information. ${ }^{41}$

Lower mental reserve and higher functional adequacy of the resected tissue preclude surgical feasibility. ${ }^{42,43}$

\section{Psychiatric assessment}

It is recommended that the presurgical evaluation should include a thorough psychiatric assessment. ${ }^{29}$ Psychiatric disorders are prevalent in epilepsy patients, and psychopathology is common in patients with TLE. Appropriate assessment can also help to anticipate acute anxiety, delusions, and other symptoms that might be aggravated in some temporal epilepsy cases, especially in the perioperative period. In addition, a history of psychiatric disorder is associated with the worst postoperative seizure outcome, although the existence of stable psychiatric disorder does not preclude epilepsy surgery. ${ }^{44}$ Psychiatric assessment should include four domains: behavioural, psychiatric, self-esteem profile, and quality of life.

\section{Epilepsy surgery}

The decision to proceed with surgical intervention is usually made by consensus agreement among the investigating clinicians. The decision is based on a rational estimation of the precision of the EZ (thus the success rate of seizure cure) and the risk-benefit analysis of the potential postoperative outcomes.

In general, outcomes are more favourable for lesional epileptic syndrome with concordance of investigation results and neuropsychological proof of "absence" of important cognitive function within the areas to be resected. In contrast, lack of concordance or evidence of important function in the pathological substrate will preclude surgical feasibility. In addition to the disease factor, patient factors such as seizure frequency, duration of illness, and co-morbidity govern the outcome. ${ }^{45}$

Conventionally, outcomes after epilepsy surgery are categorised into four classes as described by Engel. ${ }^{46}$ Epilepsy surgery is typically considered either curative, palliative, or modulatory.

Curative resective surgery involves temporal lobe surgery and extratemporal lobe surgery. Among the different epileptic syndromes, mesial temporal sclerosis has the most favourable outcomes. After curative resective surgery, outcomes in $70 \%$ of patients are reported as Engel Class I. ${ }^{47,48}$

Palliative disconnection surgery includes corpus callosotomy, hemispherectomy (anatomical/ functional), hemispherotomy, or multiple subpial transections. All of these procedures are commonly performed in paediatric patients with epilepsy and have been shown to reduce seizure frequency by $40 \%$ to $50 \%{ }^{49}$

Modulatory surgery includes gamma knife radiosurgery, vague nerve stimulation, and deep brain stimulation. Gamma knife radiosurgery has been shown to be effective in mesial temporal sclerosis patients. Seizure control is typically achieved about 10 months after radiation. Studies have shown the effectiveness of gamma knife radiosurgery in seizure control in patients with central region cavernomas and hypothalamic haemartomas. ${ }^{50}$ Vagal nerve stimulation is indicated for adults and adolescents aged $>12$ years with medically intractable partial seizures who are not candidates for potentially curative surgical resections. About $30 \%$ to $40 \%$ of patients have seizure reduction of $\geq 50 \%{ }^{51}$ Deep brain stimulation targets include the anterior nucleus and the centromedian nucleus of the thalamus, the subthalamic nucleus, the caudate nucleus, the hippocampus and the cerebellum. ${ }^{51}$ By 2 years, a median 56\% reduction in seizure frequency was observed after stimulation of anterior nucleus of the thalamus. Among the patient study group, 54\% had at least a $50 \%$ reduction in seizures, and 14 patients had seizure-free status for at least 6 months. ${ }^{52}$

Newer treatment entities include responsive or closed-loop cortical stimulation for patients with bitemporal lobe epilepsy or epilepsy originating from the eloquent cortex. The seizure reduction rate for these treatments is $>50 \%$ at 12 weeks in approximately $30 \%$ of patients. ${ }^{51}$

\section{Long-term outcomes after epilepsy surgery}

Epilepsy surgery for TLE is usually recommended because of the promising results. One study from Wiebe et al showed that, for TLE patients, surgical 
treatment resulted in significantly higher seizure freedom (58\%) than did medical treatment (8\%) at 1-year follow-up. ${ }^{47}$ The Early Randomized Surgical Epilepsy Trial in 38 patients showed that 11 of 15 patients who received surgical treatment were seizure free at 2-year follow-up compared with 0 of 23 patients who received medical treatment. ${ }^{53}$ Another study including more than 3000 patients from Germany concluded that there is an increasing trend the number of patients with non-lesional epilepsy requiring intracranial recordings. ${ }^{54}$

\section{Treatment considerations for non- lesional epilepsy}

There is always difficulty in identification of the EZ in non-lesional neocortical epilepsy. Seizure-free outcomes have been reported in $55 \%$ of patients with non-lesional TLE and in 43\% of patients with nonlesional extratemporal lobe epilepsy. ${ }^{55}$ Concordance with two or more presurgical evaluations, including interictal EEG, ictal EEG, 18-fluorodeoxyglucose positron emission tomography, or ictal single photon emission computed tomography, is significantly related to a seizure-free outcome. ${ }^{56}$ Another study showed that 9 out of 24 patients with non-lesional extratemporal epilepsy (38\%) had Engel class I outcome at a mean follow-up time of 9 years. ${ }^{57}$ In patients with TLE with MRI-negative and positron emission tomography-positive findings, surgery could achieve Engel Class I surgical outcomes at postoperative 2 years in about $79.2 \%{ }^{58}$

\section{Factors related to epilepsy surgery failure}

Epilepsy surgery failure may be caused by incorrect localisation of the EZ, very widespread EZs, or very limited resection of the suspected EZ.

After mesial temporal resection, patients may experience seizures arising from neocortical regions instead of from the residual hippocampal structure. This may imply the existence of regional epileptogenicity. The hippocampus represents the area of cortex with the lowest threshold for seizure generation and any surrounding neocortical tissue also exhibiting epileptogenicity then becomes the site of ictal onset. About $25 \%$ of patients with seizure relapse after mesial temporal sclerosis may have seizure onset in the contralateral temporal region. ${ }^{59}$

Extensive re-evaluation of patients after epilepsy surgery failure is recommended, for consideration of reoperation if the EZ can be localised.

\section{Recent advances in epilepsy monitoring and surgery}

Current applications of EEG recordings are not limited to scalp EEG and intracranial EEG with subdural electrodes and depth electrodes. Minimally invasive intracranial endovascular EEG monitoring by means of nanowire, catheter, and stent-electrode recordings is evolving. ${ }^{60}$

Stereo EEG is gaining popularity owing to its ability to make precise recordings from deep cortical areas in bilateral and multiple lobes without subjecting the patient to bilateral large craniotomies. Stereo EEG has been shown to be a useful and relatively safe tool to localise the EZ, with a procedure-related morbidity as low as 5.6\% ${ }^{61}$ Other centres have incorporated stereo EEG into a neurorobotic surgical system with comparable results. ${ }^{62,63}$

High-frequency oscillations are a potential marker for epileptogenicity and a predictive factor for positive epilepsy surgery outcomes. ${ }^{64} \mathrm{~A}$ meta-analysis has shown a small but significant relationship between the removal of the highfrequency oscillation-generating brain region and favourable outcomes. ${ }^{65}$

\section{Conclusions}

The prerequisites of seizure origin in a wellcircumscribed area of the brain and precise localisation of the EZ make modern epilepsy surgery a promising treatment modality for refractory epilepsy.

The presurgical assessment, which includes multiple disciplines, however, should be focused on two important aspects:

1. Data concordance: The individual seizure pattern is ascribed to the hypothetical brain lesions, as suggested by neurophysiological and radiological data.

2. Functional reserve: The brain pathological region, if being resected, will not leave the patient with significant morbidities.

The broad range of available diagnostic tests and surgical techniques has widened the applicability of surgical treatment. The success rates of these surgical interventions range depend strongly on different scenarios, ranging from seizure reductions of $10 \%$ to $20 \%$ to seizure-free outcomes in $>70 \%$ of patients.

Epilepsy surgery for DRE involves close collaboration and cooperation by a multidisciplinary team. Epilepsy surgery can be performed in different epilepsy centres. Patients should be referred early in their refractory disease course to a higher-level epilepsy centre for evaluation of the complex surgical options.

\section{Author contributions}

All authors have made substantial contributions to the concept of this study; acquisition of data; analysis or interpretation of 
data; drafting of the article; and critical revision for important intellectual content.

\section{Acknowledgement}

This project was supported in part by an unrestricted grant of the Hong Kong Epilepsy Society.

\section{Declaration}

All authors have disclosed no conflicts of interest. All authors had full access to the data, contributed to the study, approved the final version for publication, and take responsibility for its accuracy and integrity.

\section{References}

1. Kwan P, Arzimanoglou A, Berg AT, et al. Definition of drug resistant epilepsy: consensus proposal by the ad hoc task force of the ILAE Commission on Therapeutic Strategies. Epilepsia 2010;51:1069-77.

2. Kwan P, Brodie MJ. Definition of refractory epilepsy: defining the indefinable? Lancet Neurology 2010;9:27-9.

3. Hui AC, Kwan P. Epilepsy in Hong Kong: a literature review. Hong Kong Med J 2004;10:185-9.

4. Fong GC, Kwan P, Hui AC, Lui CH, Fong JK, Wong V. An epidemiological study of epilepsy in Hong Kong SAR, China. Seizure 2008;17:457-64.

5. Fiest KM, Sauro KM, Wiebe S, Patten SB, Kwon S, Dykeman J, Pringsheim T, Lorenzetti DL, Jette N. Prevalence and incidence of epilepsy: A systemic review and meta-analysis of international studies. Neurology 2017;88:296-303.

6. Helmers SL, Thurman DJ, Durgin TL, Pai AK, Faught E. Descriptive epidemiology of epilepsy in the U.S. population: A different approach. Epilepsia 2015;56:942-8.

7. Brodie MJ, Kwan P. Staged approach to epilepsy management. Neurology 2002;58(8 Suppl 5):S2-8.

8. Pitkänen A, Sutula TP. Is epilepsy a progressive disorder? Prospects for new therapeutic approaches in temporallobe epilepsy. Lancet Neurol 2002;1:173-81.

9. Holmes GL, Ben-Ari Y. Seizing hold of seizures. Nat Med 2003;9:994-6.

10. Bragin A, Engel J Jr, Wilson CL, Fried I, Mathern GW. Hippocampal and entorhinal cortex high-frequency oscillations (100-500 Hz) in human epileptic brain and in kainic acid-treated rats with chronic seizures. Epilepsia 1990;40:127-37.

11. Stefan H, Lopes da Silva FH. Epileptic neuronal networks: methods of identification and clinical relevance. Front Neurol 2013;4:8.

12. Choi H, Hayat MJ, Zhang R, et al. Drug-resistant epilepsy in adults: outcome trajectories after failure of two medications. Epilepsia 2016;57:1152-60.

13. Tomson T. Mortality in epilepsy. J Neurol 2000;247:15-21.

14. Sperling MR, Feldman H, Kinman J, Liporace JD, O'Connor MJ. Seizure control and mortality in epilepsy. Ann Neurol 1999;46:45-50.

15. Shackleton DP, Westendorp RG, Kasteleijn-Nolst Trenité DG, de Craen AJ, Vandenbroucke JP. Survival of patients with epilepsy: an estimate of the mortality risk. Epilepsia 2002;43:445-50.

16. Luoni C, Bisuli F, Canevini MP, et al. Determinants of health-related quality of life in pharmacoresistant epilepsy: results from a large multicenter study of consecutively enrolled patients using validated quantitative assessment.
Epilepsia 2011;52:2181-91.

17. Asadi-Pooya AA, Tajvarpour M, Vedadinezhad B, Emami M. Comparison of temporal lobe epilepsy with hippocampal sclerosis and temporal lobe epilepsies due to other etiologies. Med J Islamic Repub Iran 2015;29:263.

18. Spencer SS. When should temporal-lobe epilepsy be treated surgically? Lancet Neurol 2002;1:375-82.

19. Cascino GD. From the American Epilepsy Society 2009 Annual Course Non substrate-directed epilepsy and surgery: PRO and CON. Epilepsy Behav 2011;20:190-3.

20. Smith D, Defalla BA, Chadwick DW. The misdiagnosis of epilepsy and the management of refractory epilepsy in a specialist clinic. QJM 1999;92:15-23.

21. Perucca E, Gram L, Avanzini G, Dulac O. Antiepileptic drugs as a cause of worsening seizures. Epilepsia 1998;39:517.

22. Cramer JA, Glassman M, Rienzi V. The relationship between poor medication compliance and seizures. Epilepsy Behav 2002;3:338-42.

23. Trinka E, Dubeau F, Andermann F, et al. Successful epilepsy surgery in catastrophic postencephalitic epilepsy. Neurology 2000;54:2170-3.

24. Tonini C, Beghi E, Berg AT, et al. Predictors of epilepsy surgery outcome: a meta-analysis. Epilepsy Res 2004;62:7587.

25. Ergene E, Shih JJ, Blum DE, So NK. Frequency of bilateral independent interictal epileptiform discharges in temporal lobe epilepsy. Epilepsia 2000;41:213-8.

26. Gambardella A, Palmini A, Andermann F, et al. Usefulness of focal rhythmic discharges on scalp EEG of patients with focal cortical dysplasia and intractable epilepsy. Electroencephalogr Clin Neurophysiol 1996;98:243-9.

27. Rosenow F, Lüders H. Presurgical evaluation of epilepsy. Brain 2001;124:1683-700.

28. Macrodimitris S, Sherman EM, Forde S, et al. Psychiatric outcomes of epilepsy surgery: a systemic review. Epilepsia 2011;52:880-90.

29. Jayakar P. Invasive EEG monitoring in children; when, where and what? J Clin Neurophysiol 1999;16:408-18.

30. Cascino GD, Jack CR Jr, Parisi JE, et al. Magnetic resonance imaging-based volume studies in temporal lobe epilepsy: pathological correlations. Ann Neurol 1991;30:31-6.

31. Cendes F, Andermann F, Glorr P, et al. MRI volumetric measurement of amygdala and hippocampus in temporal lobe epilepsy. Neurology 1993;43:719-25.

32. Bae YS, Kang HC, Kim HD, Kim SH. New classification of focal cortical dysplasia: application to practical diagnosis. J Epilepsy Res 2012;2:38-42.

33. Knake S, Triantafyllou C, Wald LL, et al. 3T phased array MRI improves the presurgical evaluation in focal epilepsies: a prospective study. Neurology 2005;65:1026-31.

34. Hiratsuka Y, Miki H, Kikuchi K, et al. Sensitivity of an eight-element phased array coil in 3 Tesla MR imaging: a basic analysis. Magn Reson Med Sci 2007;6:177-81.

35. Keller SS, Glenn GR, Weber B, et al. Preoperative automated fibre quantification predicts postoperative seizure outcome in temporal lobe epilepsy. Brain 2017;140:68-82.

36. Mamelak AN, Lopez N, Akhtari M, Sutherling WW. Magnetoencephalography-directed surgery in patients with neocortical epilepsy. J Neurosurg 2002;97:865-73.

37. Papanicolaou AC, Simos PG, Castillo EM, et al. Magnetocephalography: a noninvasive alternative to the Wada procedure. J Neurosurg 2004;100:867-76. 
38. Mäkelä JP, Forss N, Jääskeläinen J, Kirveskari E, Korvenoja A, Paetau R. Magnetoencephalography in neurosurgery. Neurosurgery 2006;59:493-511.

39. Meador KJ, Loring DW. The Wada test: controversies, concerns, and insights. Neurology 1999;52:1535-6.

40. Chan S, Andrew A, Roberts D, et al. Wada memory performance does not predict memory and seizure outcome after epileptic surgery. Neurol 2017;88(16 Suppl):229.

41. Wagner K, Hader C, Metternich B, Buschmann F, Schwarzwald R, Schulze-Bonhage A. Who needs a Wada test? Present clinical indications for amobarbital procedures. J Neurol Neurosurg Psychiatry 2012;83:5039.

42. Luders HO. Textbook of Epilepsy Surgery. London: Informa; 2008.

43. Schwartz TH, Jeha LE, Tanner A, Bingaman W, Sperling MR. Late seizures in patients initially seizure free after epilepsy surgery. Epilepsia 2006;47:567-73.

44. Kanner AM, Byrne R, Chicharro A, Wuu J, Frey M. A lifetime psychiatric history predicts a worse seizure outcome following temporal lobectomy. Neurology 2009;72:793-9.

45. Téllez-Zenteno JF, Dhar R, Wiebe S. Long-term seizure outcomes following epilepsy surgery: a systematic review and meta-analysis. Brain 2005;128:1188-98.

46. Engel J Jr. Surgical Treatment of the Epilepsies. New York: Raven Press; 1993.

47. Wiebe S. Blume WT, Girvin JP, et al. A randomized, controlled trial of surgery for temporal-lobe epilepsy. $\mathrm{N}$ Engl J Med 2001;345:311-8.

48. Engel J Jr. The timing of surgical intervention for mesial temporal lobe epilepsy: a plan for a randomized clinical trial. Arch Neurol 1999;56:1338-41.

49. Malaysian Society of Neurosciences. Consensus guidelines on the management of epilepsy 2010. Available from: http://www.neuro.org.my/MSN_GUIDELINE/MSN_ GUIDELINE_Consensus\%20Guidelines\%20on\%20the\%20 Management\%20of\%20Epilepsy\%202010.pdf Accessed 15 Apr 2018.

50. Noacthar S, Borggraefe I. Epilepsy surgery: a critical review. Epilepsy Behav 2009;15:66-72.

51. Rolston JD, Englot DJ, Wang DD, Shih T, Chang EF. Comparison of seizure control outcomes and the safety of vagus nerve, thalamic deep brain, and responsive neurostimulation: evidence from randomized controlled trials. Neurosurg Focus 2012;32:E14.
52. Fisher R, Salanova V, Witt T, et al. Electrical stimulation of the anterior nucleus of thalamus for treatment of refractory epilepsy. Epilepsia 2010;51:899-908.

53. Engel J Jr, McDermott MP, Wiebe S, et al. Early surgical therapy for drug-resistant temporal lobe epilepsy: a randomized trial. JAMA 2012;307:922-30.

54. Cloppenborg T, May TW, Blümcke I, et al. Trends in epilepsy surgery: stable surgical numbers despite increasing presurgical volumes. J Neurol Neurosurg Psychiatry 2016;87:1322-9.

55. Lee SK. Surgical approaches in nonlesional neocortical epilepsy. J Epilepsy Res 2011;1:47-51.

56. Lee SK, Lee SY, Kim KK et al. Surgical outcome and prognostic factors of cryptogenic neocortical epilepsy. Ann Neurol 2005;58:525-32.

57. Noe K, Sulc V, Wong-Kisiel L, et al. Long-term outcomes after nonlesional extratemporal lobe epilepsy surgery. JAMA Neurol 2013;70:1003-8.

58. Capraz IY, Kurt G, Akdemir Ö, et al. Surgical outcome in patients with MRI-negative, PET-positive temporal lobe epilepsy. Seizure 2015;29:63-8.

59. Hennessy MJ, Elwes RD, Binnie CD, Polkey CE. Failed surgery for epilepsy: A study of persistence and recurrence of seizures following temporal resection. Brain 2000;123:2445-66.

60. Sefcik RK, Opie NL, John SE, Kellner CP, Mocco J, Oxley TJ. The evolution of endovascular electroencephalography: historical perspective and future applications. Neurosurg Focus 2016;40:E7.

61. Cossu M, Cardinale F, Castana L, et al. Stereoelectroencephalography in the presurgical evaluation of focal epilepsy: a retrospective analysis of 215 procedures. Neurosurgery 2005;57:706-18.

62. González-Martínez J, Bulacio J, Thompson S, et al. Technique, results, and complications related to robotassisted stereoelectroencephalography. Neurosurgery 2016;78:169-80.

63. Cardinale F, Rizzi M, d'Orio P, et al. A new tool for touchfree patient registration for robot-assisted intracranial surgery: application accuracy from a phantom study and a retrospective surgical series. Neurosurg Focus 2017;42:E8.

64. Gloss D, Nevitt SJ, Staba R. The role of high-frequency oscillations in epilepsy surgery planning. Cochrane Database Syst Rev 2017;(10):CD010235.

65. Holler Y, Kutil R, Klaffenböck L, et al. High-frequency oscillations in epilepsy and surgical outcome. A metaanalysis. Front Hum Neurosci 2015;9:574. 